\title{
Evolução da inteligência competitiva com base em estudo métrico de sua literatura
}

\section{Renata Cristina Teixeira}

Analista de Inteligência Competitiva da Gerência de Estratégia Tecnológica da PETROBRAS. Doutoranda em Planejamento Energético pela COPPE/UFRJ. Mestre em Tecnologia pelo CEFET/RJ. Possui o Competitive Intelligence Professional Certificate pela Fuld-Gilad-Herring Academy of Competitive Intelligence), MBA em Gestão de Negócios e Inteligência Competitiva pela UNIRIO, Especialização em Organização da Informação em Contexto Digital pela ECII/UFMG e Graduação em Biblioteconomia também pela ECI/UFMG.

Cristina Souza

\begin{abstract}
Doutora e Mestre em Engenharia de Produção pela COPPE/UFRJ. Professora Associada e substituta do Diretor de Pesquisa e PósGraduação do CEFET/RJ. Foi Coordenadora do Programa de Pós-Graduação Stricto Sensu em Tecnologia, Chefe de Departamento e Coordenadora do curso de graduação em Engenharia de Produção. Líder do grupo de pesquisa Gestão da Tecnologia.
\end{abstract}

http://dx.doi.org/10.1590/1981-5344/2037

O presente trabalho analisa as discussões promovidas sobre o tema Inteligência Competitiva (IC) no período de 1984 a 2013. Organizado na forma de pesquisa métrica, foram analisados dados bibliográficos de 3.453 artigos indexados na base de dados Scopus, identificando os assuntos que receberam maior atenção por parte de seus autores, assim como sua distribuição geográfica e temporal. Observou-se uma mudança natural nas discussões, que inicialmente concentravam-se na busca do entendimento conceitual e da justificativa da existência da IC, para um maior foco em artefatos tecnológicos capazes de proporcionar melhoramentos na sua prática. A discussão sobre ética, por sua vez, perpassa a literatura sobre IC durante todo o período estudado. Verificou-se ainda que a produção na área de Gestão tem recebido grande atenção em todas as regiões geográficas, seguida 
da área de Planejamento Estratégico, que apenas perde a segunda posição na Ásia, onde a Modelagem Matemática tem sido o principal tópico.

Palavras-chave: Inteligência competitiva; Estudo métrico; Cientometria; Bibliometria.

\section{Evolution of the competitive intelligence based on metric study of its literature}

This paper analyzes the discussions held on the theme Competitive Intelligence (CI) from 1984 to 2013. Organized in the form of a metric research, the bibliographic data of 3453 articles indexed in the Scopus database were analyzed and then it was identified which subjects received greater attention by their authors, as well as the geographical and temporal distribution of articles. The discussions started pointing to the pursuit of conceptual understanding and justification of the existence of competitive intelligence and afterward a greater focus to technological artifacts that provide improvements in the implementation of the subject as given. The Management area has received the prime attention from all the regions and the Strategic Planning is the most recurring issue in all regions but Asia, where Mathematical Models has been the main topic. The discussion on ethics pervades the literature throughout the period studied.

Key-words: Competitive intelligence; Metric research; Scientometric research; Bibliometric research

Recebido em 17.03.2015 Aceito em 15.02.2017

\section{Introdução}

O processo de globalização, o desenvolvimento de novas tecnologias e a demanda pela chamada inovação aberta têm promovido mudanças no ambiente de negócios, aumentando a necessidade de monitoramento dos concorrentes e do ambiente externo às organizações (VEUGELERS; BURY; VIAENE, 2010; PORTER; NEWMAN, 2011). Diante desse cenário, as empresas têm, cada vez mais, feito uso da Inteligência Competitiva (IC) 
(SAAYMAN et al., 2008; ZANGOUEINEZHAD; MOSHABAKI, 2009; CEKULS, 2010; TSITOURA; STEPHENS, 2012; PERUCCHI; ARAÚJO JÚNIOR, 2012).

A IC consiste em um conjunto de técnicas para a produção de conhecimento sobre mercados, competidores, produtos, tecnologias e diversos outros pontos de interesse, que nascem a partir da necessidade de otimização de resultados por parte das empresas. A IC rastreia as atividades dos concorrentes em uma ampla gama de áreas, atuando como um radar, capaz de detectar novas oportunidades ou ameaças, e permitindo a tomadores de decisão o conhecimento de informações que $a$ priori parecem insignificantes (HERRING, 1997; WANDERLEY, 2004; FULD, 2006; LODI, 2006; MILLER, 2006; QUEYRAS; QUONIAM 2006). É importante mencionar que a IC não deve ser confundida com espionagem, uma vez que não envolve práticas ilegais e antiéticas (MYBURGH, 2004; COLAKOGLU, 2011). Ao contrário da espionagem, a IC é baseada em procedimentos legais associados a um código de ética (RICHARDSON; LUCHSINGER, 2007).

Gomes e Braga (2006) contam que a origem da IC se deu com o término da Guerra Fria quando os serviços de espionagem perderam espaço. A partir desse momento, aqueles que exerciam essa função passaram a utilizar, porém de forma lícita e correta, seus conhecimentos de coleta e tratamento da informação para proporcionar vantagem competitiva às empresas. Desde então, a IC vem experimentando um grande crescimento, transformando-se em uma prática internacionalmente reconhecida (TEO; $\mathrm{CHOO}, 2001$ ).

A IC, portanto, é uma técnica que nasceu da busca por maior competitividade no ambiente de negócios. No decorrer do tempo, essa técnica passou a incorporar novas formas de trabalho, novos conhecimentos, novos processos e novas competências, passando a se expandir e se adequar a novos mercados e diferentes tipos de organização. Para o sucesso da IC, o envolvimento das pessoas se configura como um elemento-chave. Trata-se de um processo que deriva, tanto do esforço individual, quanto da utilização de redes de inteligência, sendo que essa última constitui uma das formas mais eficaz para alcançar bons resultados no processo de coleta e acesso a informações sobre o ambiente externo (WANDERLEY, 2004).

Myburgh (2004) reporta que existem várias definições para a IC, mas que todas são baseadas na seleção, coleta, processamento, interpretação, análise e distribuição de informações sobre a concorrência e o ambiente externo do negócio. A autora acrescenta que a IC é, ao mesmo tempo, um produto e um processo. É um produto, pois trata de informação acionável como base para ações específicas. É um processo enquanto atividade sistemática de aquisição, análise e avaliação de informações para obtenção de vantagem competitiva. A IC é baseada no conceito de inteligência, que é diferente da simples coleta de informações, uma vez que demanda um processo de análise em cima dos dados e informações coletados. Essa análise visa, justamente, extrair algum significado que possa ser útil para os tomadores de decisão (BOSE, 2008). 
O escopo da IC abrange quatro tipos de inteligência (DESCHAMPS; NAYAK, 1995; ROUACH; SANTI, 2001): inteligência de mercado; inteligência da concorrência; inteligência tecnológica; e inteligência estratégica e social. A inteligência de mercado tem como objetivo mapear as tendências dos consumidores, buscando identificar suas necessidades e preferências, em busca de novas oportunidades de mercado. A inteligência da concorrência pretende acompanhar a evolução das estratégias dos demais competidores ao longo do tempo e as ameaças do surgimento de produtos substitutos e de novos entrantes na indústria. A inteligência tecnológica se preocupa com a relação custo/benefício das tecnologias atuais e futuras, além de tentar prever descontinuidades tecnológicas. A inteligência estratégica e social inclui a monitoração de fatores como regulamentação, questões financeiras, fiscais, econômicas e políticas, bem como aspectos relacionados aos recursos humanos e sociais.

Além das empresas, os governos também têm usado a IC para melhor compreensão das tendências e redução de riscos associados à mudança tecnológica. Através da IC, os governos podem melhor direcionar os investimentos em pesquisa bem como subsidiar a definição de políticas mais adequadas (CALOF; SMITH, 2010). Gao et al. (2013) também reforçam a importância de monitorar os estágios do ciclo de vida das tecnologias, a fim de permitir decisões mais acertadas em relação às estratégias tecnológicas a serem adotadas.

A explosão da Internet tem contribuído grandemente para a disseminação da IC, ampliando e facilitando o acesso aos dados, e também, levando ao surgimento de novas fontes de informação, tais como sites de negócios, blogs e redes sociais como LinkedIn, MySpace e outros (VAUGHAN; YOU, 2008; BOSE, 2008). Como exemplo, pode ser citado o surgimento da Web 2.0, que se transformou em uma fonte de informação sobre a concorrência ao disponibilizar avaliações e comentários, feitos pelos clientes, sobre diversos produtos, prática essa que tem se tornado cada vez mais comum (XU et al., 2011). O desenvolvimento das Tecnologias de Informação e Comunicação (TIC) também forneceu poderosos recursos para a recuperação, processamento e análise de informações. As TICs permitiram significativa redução de tempo para a realização estudos de IC em função do chamado Quick Technolgy Intelligence Processes (QTIP), composto por quatro fatores: bases de dados de acesso instantâneo; softwares analíticos; automatização de rotinas; e padronização do processo de decisão (PORTER, 2005). Além da redução do tempo, as ferramentas disponíveis atualmente proporcionam a análise de grandes quantidades de dados, tarefa essa que não seria viável de ser coletada de forma manual ou processada por indivíduos sem o auxílio de recursos computacionais. Acrescenta-se ainda a capacidade de gerar representações visuais contendo informações úteis e que facilitam muito a interpretação dos resultados (PARK et al., 2013). 
Pinheiro (2005) diz que a IC é uma matéria relativamente recente, caracterizada por natureza interdisciplinar, e cuja constituição epistemológica e aplicada recorre principalmente a conhecimentos de administração, ciência da informação, ciência da computação e economia. Segundo a autora, os conceitos relativos à IC ainda estão em construção.

Dentro deste contexto, a fim de contribuir para uma melhor compreensão do assunto, o presente estudo tem como objetivo investigar a evolução da Inteligência Competitiva por meio de sua caracterização temporal, a partir da revisitação da literatura científica publicada. Baseando-se em 3.453 documentos recuperados na base de dados da Scopus em pesquisa realizada em janeiro de 2014 com limitação temporal até o ano de 2013, foram identificados: os principais temas relacionados à IC que a caracterizaram em cada década; a rede de relações entre estes temas; as grandes áreas temáticas onde a IC tem sido incorporada; a distribuição geográfica da produção do conhecimento sobre IC, com o principal foco abordado em cada continente; e, por fim, os principais autores e instituições que têm se dedicado a essa temática.

Esse estudo, portanto, apresenta um registro da memória das pesquisas sobre IC no mundo, de modo que se possa resgatar sua evolução no futuro. Essa é uma questão importante, principalmente para aqueles pesquisadores que gostam e estudam a história da ciência e da tecnologia.

O trabalho está organizado em seções. A seção 2 descreve 0 conjunto de métodos utilizados para a produção deste estudo. A seção 3 apresenta e discute os resultados. Finalmente, são feitas as considerações finais.

\section{Aspectos metodológicos}

A infometria foi a metodologia aplicada neste estudo. De acordo com Santos e Kobashi (2009), infometria é um termo que se refere às atividades relacionadas com as métricas de informação e que faz uso de métodos da bibliometria e cientometria para abordar os aspectos cognitivos da atividade científica. Os estudos de infometria visam fornecer um método preciso e presumivelmente objetivo para medir a contribuição de informações científicas para o avanço do conhecimento (YANG et al., 2013).

Para a realização desse estudo foi um levantamento de artigos indexados na Scopus, que é uma base de dados de cobertura mundial e que abrange cerca de 47 milhões de registros em áreas científicas, técnicas, médicas, sociais, artes e humanidades.

A estratégia de busca utilizada foi baseada no termo "competitive intelligence" nos campos: resumo, título e palavras-chave, sem qualquer delimitação temporal, o que resultou em 3.453 documentos datados entre 1984 e 2013. Os dados dos artigos obtidos na base de dados foram exportados para o software de mineração de textos VantagePoint, o qual 
foi utilizado para o tratamento das informações e exteriorização de padrões.

De forma complementar buscou-se fazer a leitura dos campos título, resumo, termos de indexação e título da fonte (periódico ou evento), excluindo-se apenas os registros que não possuíam resumos. A leitura de parte do documento foi feita quando os campos anteriormente citados não esclareciam completamente o propósito do artigo.

Além da análise conceitual, os artigos também foram identificados pela distribuição temporal e concentração em regiões geográficas e áreas do conhecimento.

\section{Resultados e discussão}

A Figura 1 apresenta a distribuição temporal dos registros recuperados no levantamento sobre a IC na base Scopus. É possível observar que a primeira década dos anos 2000 concentra a maioria dos artigos.

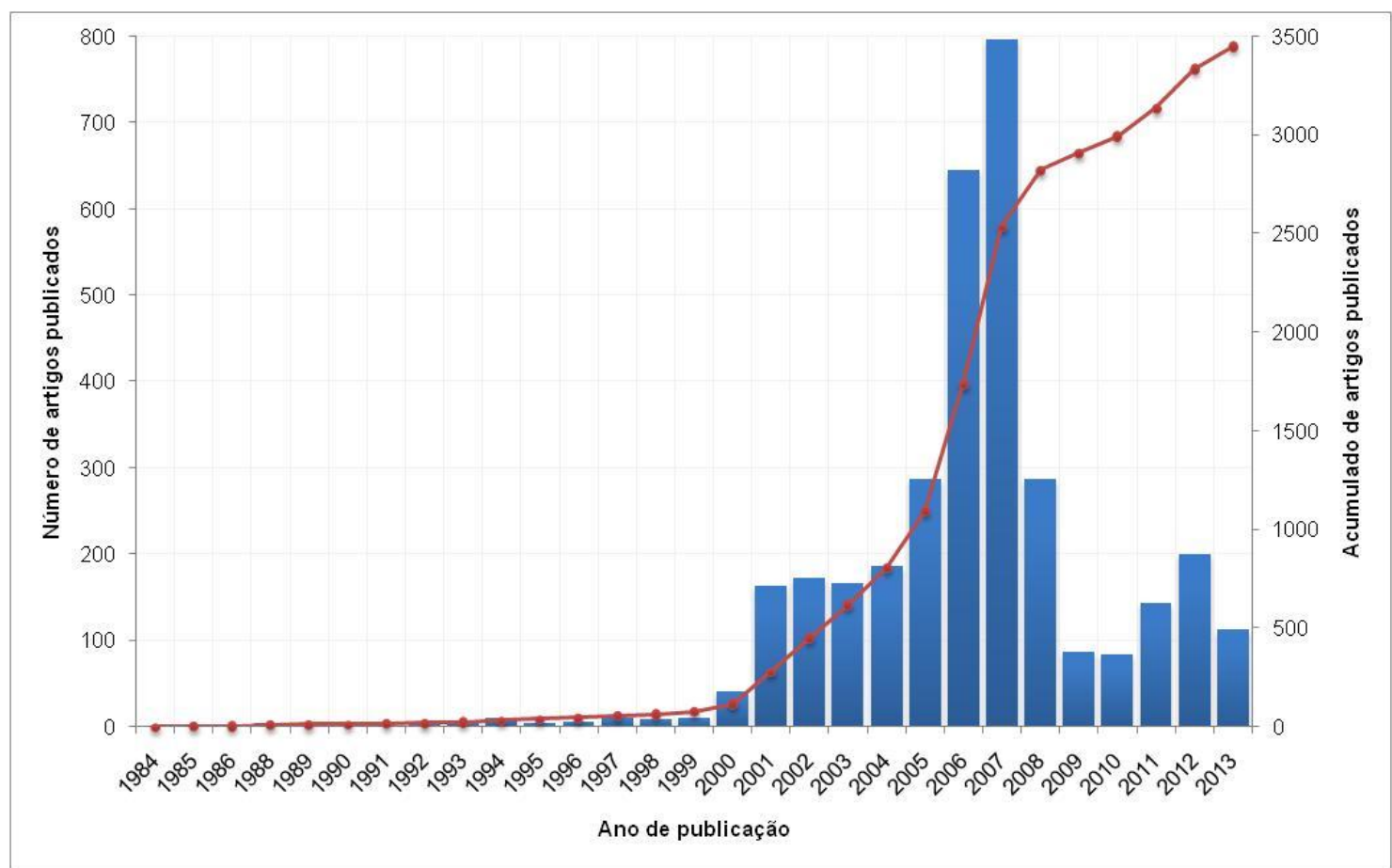

Figura 1 - Distribuição temporal e acumulado das publicações

Fonte: Elaboração própria a partir dos dados recuperados.

As primeiras publicações sobre o assunto datam dos anos 1980 e abordam o conceito de IC e sua razão de ser. Discutem ainda a busca por informação e a recuperação de documentos, além do acesso e da disseminação das informações recuperadas. No final da década, que representa $0,4 \%$ das publicações abrangidas por esse estudo, a atenção das discussões voltou-se para o ambiente competitivo do mercado e 
também para o planejamento estratégico e seus benefícios para as empresas.

Os anos 1990 concentram pouco menos de 3\% das publicações. Durante esse período, a inteligência competitiva começou a fazer uso de técnicas como análise estatística de literatura, análise de pontos cegos e benchmarking. Nessa época, também começaram as discussões sobre ferramentas colaborativas. Temas como inovação, prospecção tecnológica e inteligência tecnológica, apontando o potencial da IC para ganho de mercado a partir do desenvolvimento tecnológico, foram adicionados na discussão sobre gerenciamento estratégico.

$\mathrm{Na}$ primeira década do século XXI, concentram-se $83 \%$ dos artigos e, desse total, 31\% tratam de artefatos tecnológicos para execução ou melhoria de processos da IC.

Ferramentas como Business Intelligence (BI), datawarehouse, mineração de dados, de texto e da web, metadados e o Enterprise Resource Planning (ERP) são os principais pontos de melhorias nos processos de IC apontados na década de 2000. Seguem assuntos voltados a uma automação cada vez maior, tanto na parte de coleta de informações quanto na parte de análise, com a construção de algoritmos, inteligência artificial, web semântica, sistemas semânticos e ontologias.

Outro aspecto observado na década de 2000 foi o crescimento do interesse por redes de colaboração, sejam redes empresariais ou sociais. Também foi verificado um aumento dos estudos envolvendo redes de colaboração entre universidades e empresas, tendo como principal enfoque o viés tecnológico, com a presença de termos como informação tecnológica, inovação, educação tecnológica, propriedade intelectual, transferência de tecnologias e de conhecimento, prospecção tecnológica e enriquecimento de ideias para inovação.

Nos três primeiros anos da década de 2010, a discussão do ferramental da IC foi se tornando cada vez mais sofisticada. Business Intelligence de acesso móvel ou em nuvem, lógica fuzzy, Predictive Model Markup Language (PMML) e redes neurais tornaram-se os temas de maior interesse, além dos aspectos sócio-cognitivos e de capital humano. Esse período conta com quase $14 \%$ dos registros e, desse total, mais de $42 \%$ produziu informação sobre tecnologias para melhoria das funções exercidas pela IC.

É importante ressaltar que as discussões sobre ética perpassam todos os períodos da literatura sobre o tema, aparecendo pela primeira vez em 1986 e depois em todos os anos a partir de 1999, em conjunto com os mais diversos temas. A Figura 2 apresenta os principais termoschave que caracterizam cada década de acordo com a literatura analisada. 

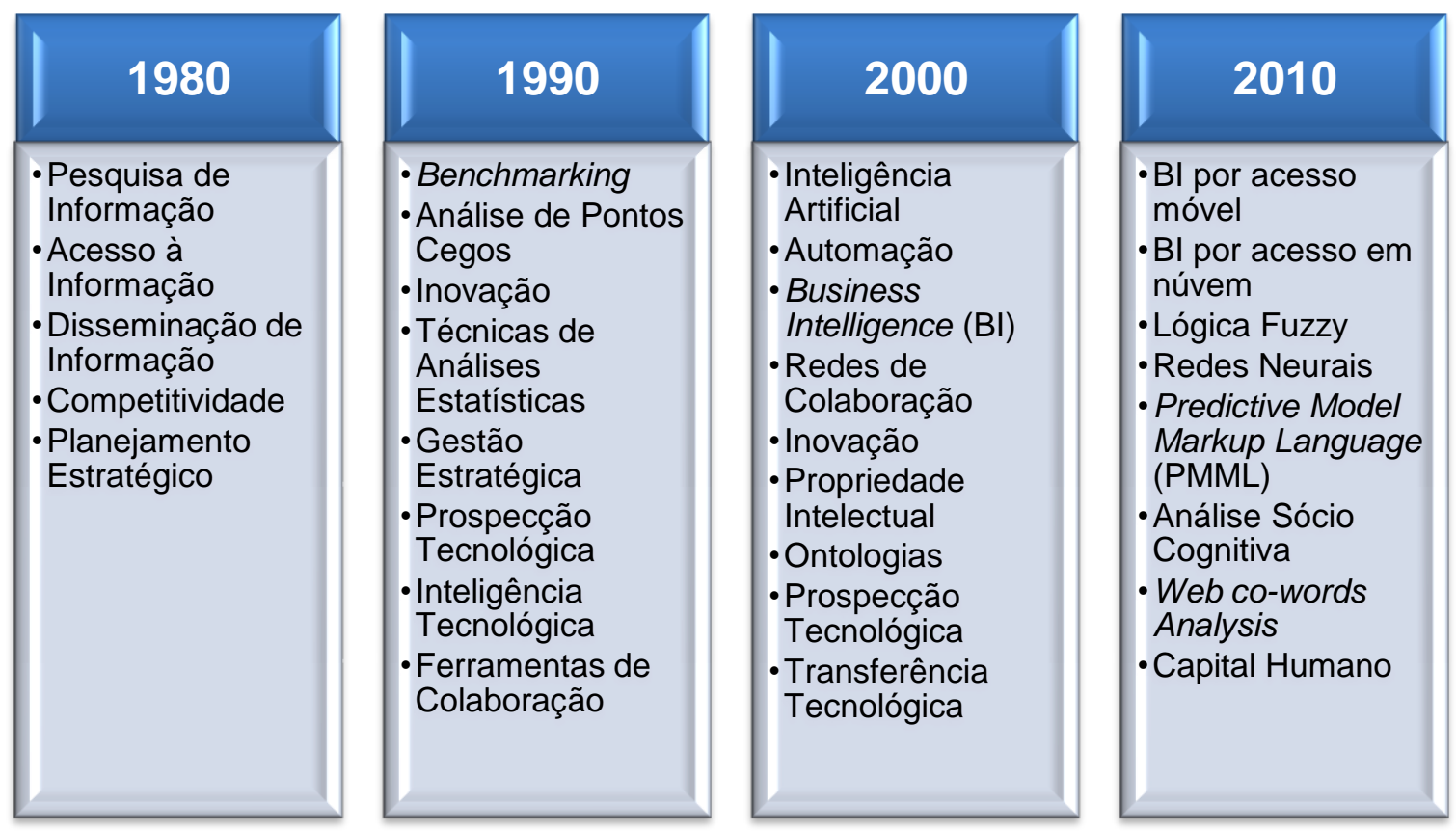

Figura 2 - Principais termos-chave que caracterizam cada década de acordo com a literatura sobre IC

Fonte: Elaboração própria a partir dos dados recuperados.

A Tabela 1, por sua vez, apresenta a listagem dos principais termoschaves considerando-se toda a literatura analisada, sem separação por décadas. Essa relação foi feita baseando-se, tanto nos termos-chave do autor (termos publicados no artigo original), quanto nos termos-chave de indexação (termos de vocabulário controlado ou thesaurus adicionados aos artigos no momento da entrada na base de dados).

\begin{tabular}{lc||lc}
\hline \hline \multicolumn{1}{c|}{ Termos-chave } & $\begin{array}{c}\text { Artigos } \\
\text { Publicados }\end{array}$ & \multicolumn{1}{c}{ Termos-chave } & $\begin{array}{c}\text { Artigos } \\
\text { Publicados }\end{array}$ \\
\hline \hline Inteligência Competitiva & 3253 & Economia Industrial & 253 \\
Planejamento Estratégico & 503 & Comércio Eletrônico & 231 \\
Gestão Industrial & 417 & Satisfação de Clientes & 226 \\
Business Intelligence & 372 & Mineração de Dados & 220 \\
Competição & 368 & Desenvolvimento de & 214 \\
Modelagem Matemática & 349 & Produtos & 208 \\
Marketing & 346 & Sistema de Informações & 183 \\
Tomada de Decisão & 338 & Gesolução de Problemas & 175 \\
Sociedades e Instituições & 325 & Gestão de Projetos & 171 \\
Tecnologias da Informação & 279 & Gestão da Informação & 154 \\
\hline
\end{tabular}

Tabela 1 - Lista dos principais termos-chave empregados no conjunto de dados recuperados.

Fonte: Elaboração própria a partir dos dados recuperados. 
Embora a listagem dos termos-chave aponte as temáticas de maior interesse, a identificação de como esses termos interagem entre si permite uma melhor compreensão do escopo das publicações conforme mostrado na Figura 3, que apresenta a correlação entre os principais termos recuperados na busca. A expressão "Competitive Intelligence" é a que possui maior número de ocorrências, bem como a que aparece mais associada aos demais termos. Tal resultado já era esperado em função da estratégia de busca adotada que foi consonante com o objetivo desse estudo. "Business Intelligence" é o segundo termo em número de ocorrências e interações. Os termos relacionados a ferramentas de tecnologia e comunicação também são muito recorrentes, o que denota grande interesse no desenvolvimento de ferramentas que propiciem maior eficiência aos processos da IC. O termo "Innovation", por exemplo, apresenta relação com termos da área gerencial como "Business Strategy", "Competition" e "Knowledge Management" e possui também uma ligação com termos do ferramental tecnológico como "Data Mining".

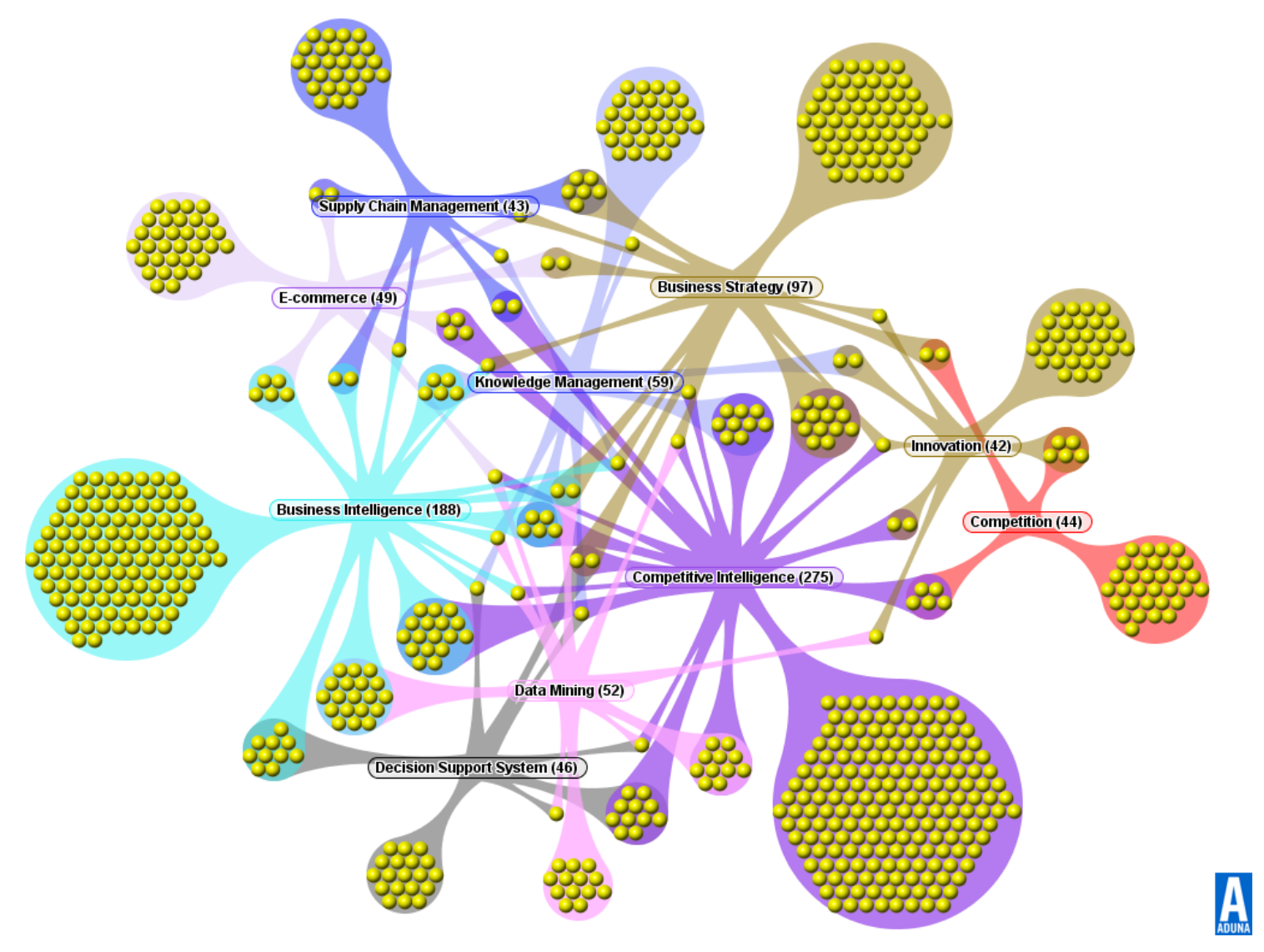

Figura 3 - Rede de relacionamentos entre os principais termos-chave Fonte: Elaboração própria a partir dos dados recuperados.

A Tabela 2 apresenta as principais áreas do conhecimento que possuem interface com a IC. Essas áreas foram definidas no momento da indexação dos artigos em sua fonte. Percebe-se que a IC é uma disciplina que perpassa áreas diversas como Ciências Sociais e Computacionais, Gestão e Materiais, Bioquímica e Matemática, ilustrando o seu caráter e 
utilidade interdisciplinares. A interdisciplinaridade, conforme apontado por Tijssen (1992), envolve o uso direto ou indireto de conhecimento, métodos, técnicas e dispositivos ou outros produtos oriundos de diversos campos do saber. Assim sendo, a interdisciplinaridade conduz a uma sinergia que resulta da atuação de pessoas com atuações diversificadas, permitindo que se antecipem problemas e se encontrem soluções sob uma perspectiva mais holística.

\begin{tabular}{l|c}
\hline \hline \multicolumn{1}{c|}{ Principais Áreas do Conhecimento } & $\begin{array}{c}\text { Artigos } \\
\text { Publicados }\end{array}$ \\
\hline \hline Engenharia & 1.655 \\
Ciência da Computação & 1.399 \\
Negócios, Gestão e Contabilidade & 793 \\
Ciências da Decisão & 422 \\
Ciências Sociais & 366 \\
Matemática & 335 \\
Engenharia Química & 152 \\
Bioquímica, Genética e Biologia Molecular & 113 \\
Ciências dos Materiais & 109 \\
Economia, Econometria e Finanças & 108 \\
\hline \hline
\end{tabular}

Tabela 2 - Principais áreas de conhecimento por onde transita a IC

Fonte: Elaboração própria a partir dos dados recuperados.

Todos os artigos com indicação de afiliação de autores foram analisados com vista a um maior entendimento da participação dessas instituições. A distribuição geográfica, considerando-se o número total de instituições com publicações sobre IC, pode ser observada no Gráfico 1. América, Europa e Ásia concentram a maioria das instituições de forma muito próxima: $34 \%, 32 \%$ e $30 \%$ respectivamente.

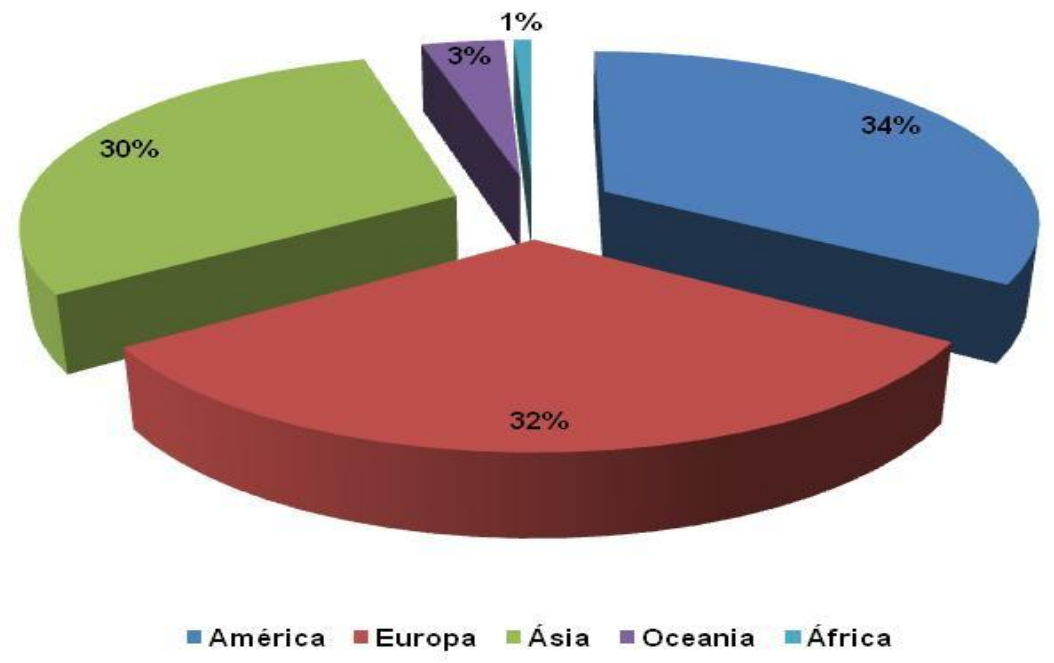

Gráfico 1 - Distribuição continental das instituições que publicaram artigos no tema

Fonte: Elaboração própria a partir dos dados recuperados. 
Dos artigos publicados no continente asiático, pouco mais de $50 \%$ são oriundos da China. Pouco mais de $82 \%$ dos artigos são de instituições de ensino e pesquisa. Dentre os $18 \%$ das publicações vinculadas a empresas, mais da metade $(60 \%)$ pertence ao continente americano, sendo a maioria localizada nos Estados Unidos e Canadá.

A Figura 4 apresenta a distribuição geográfica das publicações sobre a IC, segundo o país de origem de cada instituição. Conforme pode ser observado, os Estados Unidos lideram o ranking com 556 artigos, seguidos da China com 467, do Reino Unido com 207 e da Alemanha com 158 publicações. O Brasil aparece na $8^{\text {a }}$ colocação, com 73 artigos no tema. De acordo com os dados, $96 \%$ dos registros foi publicado em língua inglesa, seguido por 1,2\% na língua alemã.

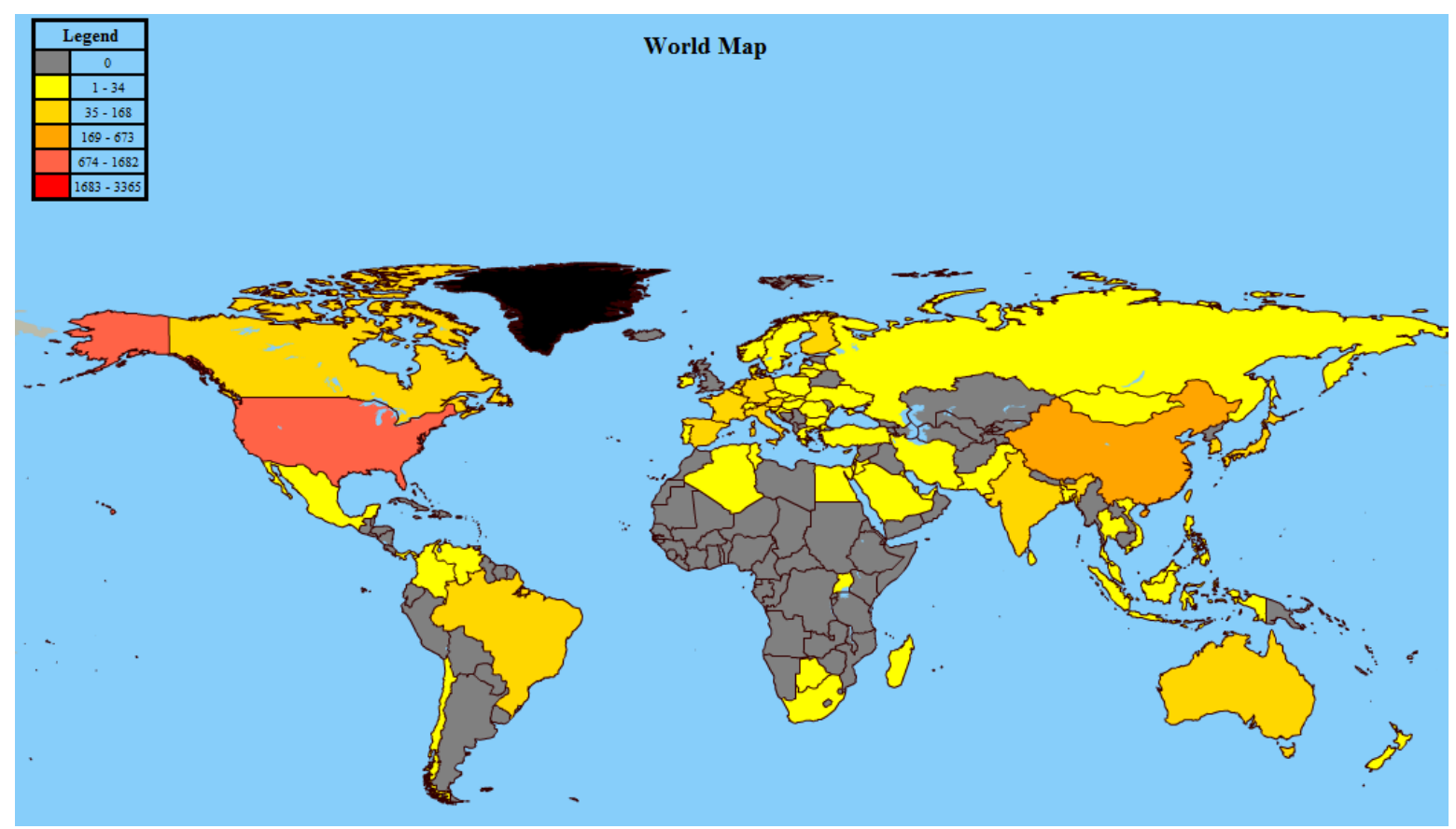

Figura 4 - Distribuição geográfica de artigos por país de origem da instituição.

Fonte: Elaboração própria a partir dos dados recuperados.

No grupo das publicações brasileiras, a discussão atenta-se principalmente para artefatos tecnológicos como ferramentas de Business Intelligence, mineração de textos, sistemas semânticos e taxonomias. Nesse grupo também foi percebida forte atenção ao perfil do profissional de IC e da formação das equipes que atuam nessa função. Uma característica marcante nas publicações brasileiras são os estudos de caso, a maioria nas áreas da inovação, engenharia, energia, nanotecnologia e saúde.

A área de gestão é a que recebe a maior atenção nas Américas, especialmente nos Estados Unidos, no Canadá e no Brasil. Os temas mais recorrentes nessa região são planejamento estratégico (18\%), marketing 
(14\%), gestão industrial (13\%), tomada de decisão (13\%) e economia industrial (10\%).

No grupo de países asiáticos, a discussão foca em modelos matemáticos $(16 \%)$, planejamento estratégico (14\%), gestão industrial $(12 \%)$ e comércio eletrônico (10\%). Nesse grupo, também se nota uma massiva participação de universidades e instituições de ensino tais como: Instituto de Tecnologia de Harbin (3,6\%), Universidade de Zhejiang $(3,1 \%)$, Universidade de Cidade de Hong Kong $(2,8 \%)$ e Universidade de Shanghai Jiaotong $(2,6 \%)$.

Universidades e instituições de ensino também têm larga participação no grupo de países europeus, especialmente no Reino Unido, na Alemanha, nos Países Baixos e na França. Os principais tópicos discutidos nos artigos europeus são planejamento estratégico $(15 \%)$, gestão industrial (13\%), modelos matemáticos (12\%) e tomada de decisão (11\%).

Austrália e Nova Zelândia representam a região da Oceania. Ao contrário das outras regiões, na Oceania se observa uma larga participação de empresas, como a New Zealand Refining Company e empresas australianas especializadas em tecnologia da informação e serviços de gestão: Distributed Systems Technology Centre, Deontik Pty Ltd e IDSM Pty Ltd. Na Oceania, os principais tópicos são planejamento estratégico $(20 \%)$, inteligência de negócios (19\%), sistemas de informação (16\%) e tomada de decisão (15\%).

O continente africano, representado nesse estudo exclusivamente pela África do Sul, também dá prioridade ao planejamento estratégico $(19 \%)$. Os tópicos competição, estratégia competitiva e países em desenvolvimento representam, cada um, 15\% das contribuições.

O Gráfico 2 apresenta a lista das principais instituições publicantes, bem como o autor com maior número de publicações em cada uma dessas instituições. Nesta figura, é possível perceber que em algumas instituições o conhecimento é bem distribuído enquanto que, em outras, pouco autores são responsáveis pelo tema na instituição. 


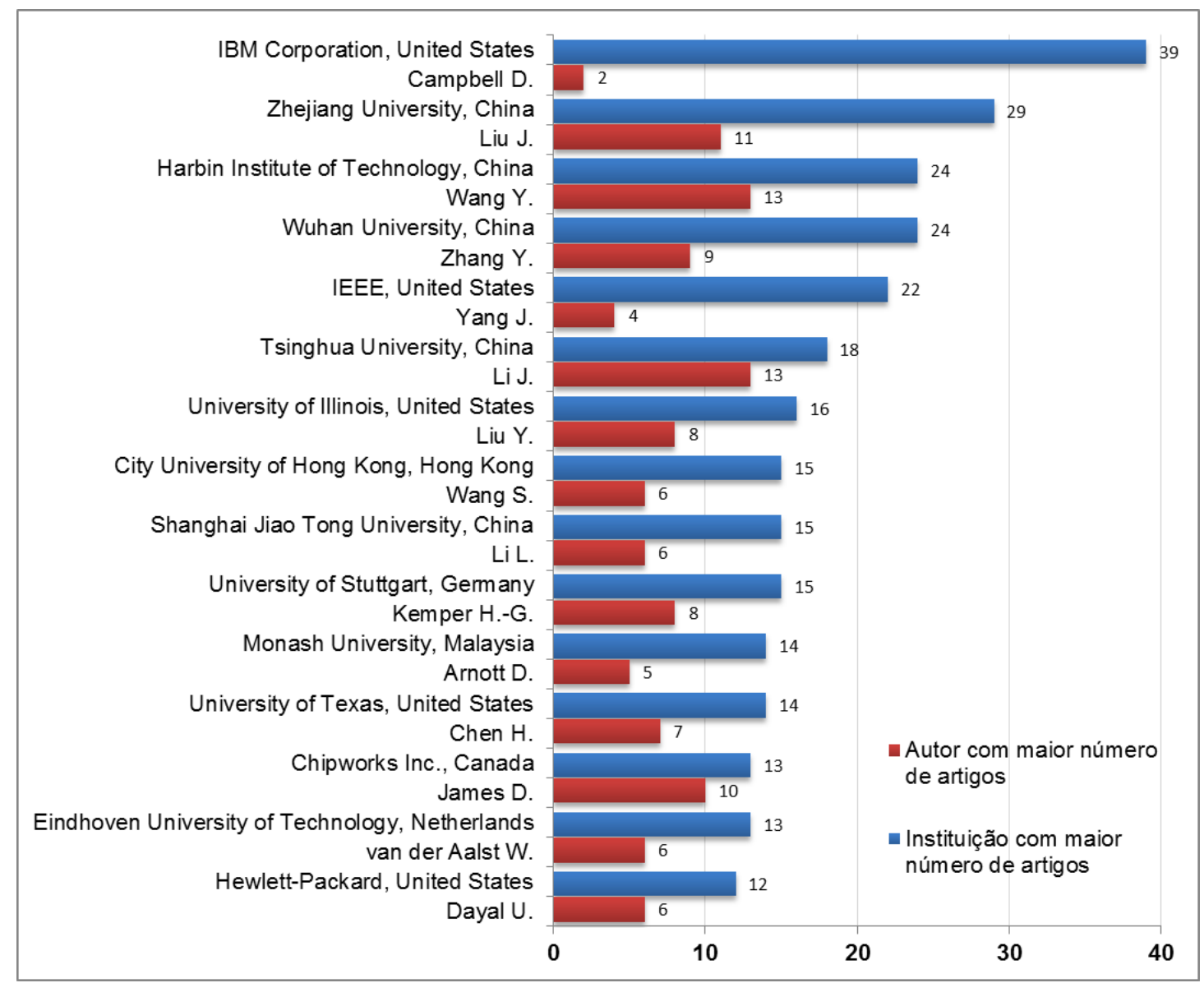

Gráfico 2 - Instituições com maior número de artigos publicados e principal autor dentro de cada instituição

Fonte: Elaboração própria a partir dos dados recuperados.

\section{Considerações finais}

A inteligência competitiva teve um período grande de discussão da sua razão de ser, com artigos apresentando vantagens dessa nova forma de trabalho, e de se relacionar com informações do mercado competidor. Ao longo do tempo, os profissionais e/ou pesquisadores da área foram trocando experiências ao descreverem suas rotinas, apontando os problemas vividos e os sucessos alcançados.

Com o passar do tempo e com a solidificação dessa metodologia de trabalho, nota-se uma mudança natural das discussões sobre a inteligência competitiva, apontando não mais para a justificação de sua existência, e sim para melhoramentos na sua execução e nos novos aparatos que propiciam esse melhoramento.

No presente estudo realizou-se a caracterização da inteligência competitiva através da análise de sua literatura, mostrando o curso de sua evolução ao longo do tempo. O estudo métrico, metodologia aplicada nesse trabalho, mostrou-se útil para analisar o desenvolvimento $\mathrm{e}$ apresentar um mapeamento da IC no mundo. 
Dada a importância dos processos de Inteligência Competitiva para os mais diversos setores públicos e privados, espera-se que esse trabalho possa contribuir para uma maior difusão da IC no ambiente acadêmico, governamental e empresarial. Acrescenta-se que o presente estudo também se configura como um registro que visa preservar a memória da IC, o que é interessante principalmente para os pesquisadores interessados na história da ciência e da tecnologia.

\section{Referências}

BOSE, R. Competitive intelligence process and tools for intelligence analysis. Industrial Management \& Data Systems, v. 108, n. 4, p. 510528, 2008.

CALOF, J.; SMITH, J. The integrative domain of foresight and competitive intelligence and its impact on R\&D management. R\&D Management, v. 40, n. 1, p. 31-39, 2010.

CEKULS, A. Competitive intelligence model in Latvian enterprises. TILTAI, v. 53, n. 4 , p. $35-45,2010$.

COLAKOGLU, $T$. The problematic of competitive intelligence: how to evaluate \& develop competitive intelligence? Procedia - Social and Behavioral Sciences, v. 24, p. 1615-1623, 2011.

DESCHAMPS, J. P.; NAYAK, P. R. Product juggernauts: how companies mobilize to generate a stream of market winners. Boston: HBS Press, 1995.

FULD, L. M. The secret language of competitive intelligence: how to see through \& stay ahead of business disruptions, distortions, rumors \& smoke screens. New York: Crown Business, 2006.

GAO, L. et al. Technology life cycle analysis method based on patent documents. Technological Forecasting and Social Change, v. 80, n. 3, p. 398-407, 2013.

GOMES, E. B. P.; BRAGA, F. R. Inteligência competitiva no Brasil: uma realidade corporativa. Puzzle. Revista de Inteligencia Competitiva, v. 6, n. 23, p. 5-10, 2006.

HERRING, J. P. Producing CTI that meets senior management's needs and expectations. In: SCIP COMPETITIVE TECHNICAL INTELLIGENCE SYMPOSIUM, 1997, Boston. Proceedings... Boston: SCIP, 1997.

LODI, C. F. G. Planejamento por cenários e inteligência competitiva: integrando seus processos para tomar decisões estratégicas mais eficazes. In: STAREC, C.; GOMES, E. B. P.; CHAVES, J. B. L. Gestão estratégica da informação e inteligência competitiva. São Paulo: Saraiva, 2005. p. 124142.

MILLER, J. P. O milênio da inteligência competitiva. Porto Alegre: Bookman, 2002. 
MYBURGH, S. Competitive intelligence: bridging organizational boundaries. Information Management Journal, v. 38, n. 2, p. 46-55, 2004.

PARK, H. et al. A patent intelligence system for strategic technology planning. Expert Systems with Applications, v. 40, n. 7, p. 2373-2390, 2013.

PERUCCHI, V.; ARAÚJO JÚNIOR, R. H. Produção científica sobre inteligência competitiva da Faculdade de Ciência da Informação da Universidade de Brasília. Perspectivas em Ciência da Informação, v. 17, n.2, p. 37-56, 2012.

PINHEIRO, L. V. R. Inteligência competitiva como disciplina da ciência da informação e sua trajetória e evolução no Brasil. In: STAREC, C.; GOMES, EL.; BEZERRA, J. Gestão estratégica da informação e inteligência competitiva. São Paulo: Saraiva, 2005, p. 17-32.

PORTER, A. L. QTIP: quick technology intelligence processes. Technological Forecasting and Social Change, v. 72, n. 9, p. 1070-1081, 2005.

PORTER, A. L.; NEWMAN, N. C. Mining external R\&D. Technovation, v. 31, n. 4, p. 171-176, 2011.

QUEYRAS, J.; QUONIAM, L. Inteligência competitiva. In: TARAPANOFF, K. (Ed.). Inteligência, informação e conhecimento em corporações. Brasília: IBICT; UNESCO, 2006. p. 73-98.

RICHARDSON, L.; LUCHSINGER, V. Strategic marketing implications in Competitive Intelligence and the economic espionage act of 1996. Journal of Global Business Issues, v. 1, n. 2, p. 41-45, 2007.

ROUACH, D.; SANTI, P. Competitive Intelligence adds value: five intelligence attitudes. European Management Journal, v. 19, n. 5, p. 552$559,2001$.

SAAYMAN, A. et al. Competitive intelligence: construct exploration, validation and equivalence. Aslib Proceedings, v. 60, n. 4, p. 383-411, 2008.

SANTOS, R. N. M.; KOBASHI, N. Y. Bibliometria, cientometria, infometria: conceitos e aplicações. Pesq. Bras. Ci. Inf., Brasília, v. 2, n. 1, p. 155-172, jan./dez. 2009.

TEO, T. S. H.; CHOO, W. Y. Assessing the impact of using the internet for competitive intelligene. Information \& Management, v. 39, n. 1, p. 67-83, 2001.

TIJSSEN, R. J. W. A quantitative assessment of interdisciplinary structures in science and technology: co-classification analysis of energy research. Research Policy, v. 21, n. 1, p. 27-34, 1992.

TSITOURA, N.; STEPHENS, D. Development and evaluation of a framework to explain causes of competitive intelligence failures. Information Research, v. 17, n. 2, 2012. Disponível em: 
<http://informationr.net/ir/17-2/paper521.html>. Acesso em: 19 maio 2013.

VAUGHAN, L.; YOU, J. Content assisted web co-link analysis for competitive intelligence. Scientometrics, v. 77, n. 3, p. 433-444, 2008.

VEUGELERS, M.; BURY, J.; VIAENE, S. Linking technology intelligence to open innovation. Technological Forecasting and Social Change, v. 77, n. 2, p. 335-343, 2010.

WANDERLEY, A. V. M. Conception et implantation d'unsystème d'intelligence compétitive dans une entreprise pétrolière dans un environnement de dé réglementation. 2004. 322f. Tese (Docteur en Sciences de I'Information et la Communication) - Université de Droit, D'Economie et de Sciences D'Aix-Marseille III, Marseille, France, 2004.

$\mathrm{XU}, \mathrm{K}$. et al. Mining comparative opinions from customer reviews for competitive intelligence. Decision Support Systems, v. 50, n. 4, p. 743754, 2011.

YANG, L. et al. Global trends of solid waste research from 1997 to 2011 by using bibliometrics analysis. Scientometrics, v. 96, n. 1, p. 133-146, 2013.

ZANGOUEINEZHAD, A.; MOSHABAKI, A. The role of structural capital on competitive intelligence. Industrial Management \& Data Systems, v. 109, n. 2, p. 262-280, 2009. 